25 (23\%) IBD nurse specialists and $5(5 \%)$ gastroenterology pharmacists, and were used for analysis.

Over half $(61,55 \%)$ only carry out TDM in non-response. The remainder use TDM routinely, during stable maintenance therapy for patients in remission. Only 15 (14\%) respondents reported being clear and confident in their understanding of the difference between drug-sensitive and drug-tolerant assays. Moreover, most $(82,75 \%)$ were unsure as to which type their laboratory uses. Lower therapeutic thresholds used by clinicians were variable (figure 1).

Consultants, high-frequency TDM users ( $>3$ requests/ month) and clinicians with larger anti-TNF cohorts (>100 patients) were significantly more likely to select the 'most appropriate' answer to at least 1 of the 5 TDM scenarios (figure 2).

Conclusions These results demonstrate marked heterogeneity in the practical use, understanding and interpretation of biologic TDM in IBD. Biologic decision-making, informed by TDM, should involve consultation with experienced clinicians who are frequent TDM users, ideally, as part of a multidisciplinary, biologics-focused IBD meeting.

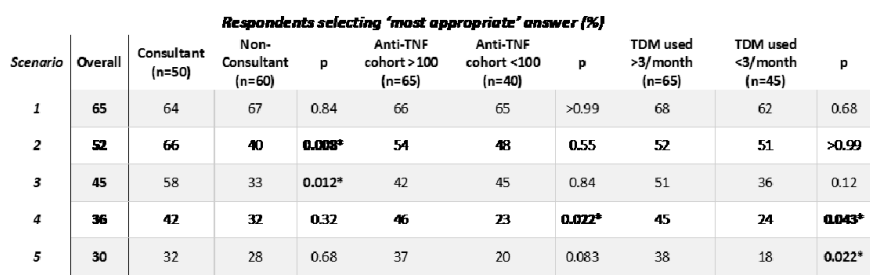

Abstract PWE-047 Figure 2 Percentage of respondents selecting the 'most appropriate' answer to each scenario, overall and after diving by subgroup (NB: 5 respondents were unsure of the size of their anti-TNF cohort and were not included in that analysis)

\section{PWE-048 THE NATURE OF CHECKPOINT INHIBITOR-ASSOCIATED LOWER GASTROINTESTINAL TOXICITY: A SINGLE CANCER CENTRE EXPERIENCE}

\footnotetext{
1,2,3 Amit Samani* ${ }^{*}$ 2,3 Zayd Tippu, ${ }^{2}$ Shuai Zhang, ${ }^{2}$ Sophie Merrick, ${ }^{1,2}$ Hajir Ibraheim, ${ }^{1,2}$ Nicholas Powell, ${ }^{1,2}$ Debra Josephs, ${ }^{1,2}{ }^{2}$ Sophie Papa. ${ }^{1}$ King's College London, London, UK; ${ }^{2}$ Guy's and St Thomas' NHS Foundation Trust, London, UK
}

\subsection{6/gutjnl-2018-BSGAbstracts. 180}

Introduction Immune checkpoint inhibitors (CPi) are a standard of care for various cancers however can cause unpredictable toxicity, notably of the lower gastrointestinal (GI) tract. As continued expansion of their use is anticipated, we sought to investigate the 'real world' frequency and characteristics of such toxicity.

Methods We conducted a retrospective case-note review of patients treated with $\geq$ one dose of either nivolumab monotherapy $(\mathrm{N})$, pembrolizumab monotherapy $(\mathrm{P})$, sequential pembrolizumab/ipilimumab (P-I) or combination nivolumab +ipilimumab $(\mathrm{n}+\mathrm{I})$ between 01/03/2016 - 28/02/ 2017. Toxicity (tox) was graded using NCI Common Terminology Criteria for Adverse Events v4.03. Statistical comparisons used Student's T-test or Chi Squared tests with Bonferroni corrections.
Results 141 patients were treated (49 female, 34.8\%), mean age of 64.6 years. 71,33 and 37 (50.3\%, 23.4\% and 26.2\%) had melanoma, lung and renal cancers respectively.

83 received $\mathrm{P}(58.9 \%)$ while $39(27.7 \%)$ received $\mathrm{N}$ with only $11(7.8 \%)$ and $8 \quad(5.7 \%)$ receiving $n+I$ or $\quad$-I respectively.

Of these, 29 had any grade GI tox. Their mean age $(65.7$ years), female proportion (34.4\%) and proportion with melanoma, lung or renal cancers $(55.2 \%, 20.1 \%$ and $24.1 \%$ respectively) did not significantly differ from the 112 patients with no GI tox.

However, $6(20.6 \%)$ vs $2(1.8 \%)$ received P-I and 5 $(17.2 \%)$ vs $6(5.4 \%)$ received $\mathrm{n}+\mathrm{I}$ in the GI and no-GI tox cohorts respectively which was significantly different $(p=0.001$ and 0.036 respectively). Only $2(7.1 \%)$ in the GI tox cohort had initial progressive disease compared to 47 (46.1\%, $\mathrm{p}<0.001)$.

In the GI tox cohort, 17 had grade 1 (G1) tox, 5 G2 and $7 \mathrm{G} 3 / 4$. When comparing the G3/4 to G1 cohort there was a trend towards younger age (54.3 vs 67.7 years), treatment with P-I or $\mathrm{n}+\mathrm{I}(71.5$ vs $17.8 \%)$ and higher CRP (100 vs $62 \mathrm{mg} / \mathrm{L})$.

The rate of G3/4 toxicity was similar to the reported literature. For n+I, $5 / 11(45.5 \%)$ had any grade GI tox with $3 / 11$ (27.3\%) having grade $3 / 4$.

Finally, of those with G3/4 tox, 4 had documented fever at presentation, only 1 had haematochezia and all had diarrhoea of $\geq 5 \mathrm{x} /$ day. All required IV methylprednisolone but 3 received alternate routes initially ( 2 oral, 1 rectal). Three patients required infliximab with only 1 requiring $\geq 1$ dose.

Conclusions A significant proportion of patients develop lower GI tox with CPi. They were more likely to have been treated with ipilimumab containing regimens and to have a favourable response to therapy although this may have been confounded by the higher use of $n+I$ in this cohort. The rate of G3/4 toxicity was similar to the literature. Some patients are initially treated with oral/rectal steroid; they may benefit from earlier IV therapy. Infliximab as a single dose is usually effective.

\section{PWE-049 ASSESSMENT OF SYMPTOMATIC HYPOPHOSPHATEMIA WITH IRON ISOMALTOSIDE IN INFLAMMATORY BOWEL DISEASE PATIENTS}

${ }^{1}$ Walter Reinisch*, ${ }^{2}$ Jens Frederik Dahlerup, ${ }^{3}$ Richard Derman. ${ }^{1}$ Medical University of Vienna, Vienna, Austria; ${ }^{2}$ Aarhus University Hospital, Aahus, Denmark; ${ }^{3}$ Thomas Jefferson University, Philadelphia, USA

\subsection{6/gutjnl-2018-BSGAbstracts. 181}

Introduction Patients with inflammatory bowel disease (IBD) often need intravenous (IV) iron but some IV irons have been associated with hypophosphatemia in as many as $47 \%-70 \%$ of patients with iron deficiency anaemia (IDA) without chronic kidney disease with severe hypophosphatemia seen in up to $13 \%$ of the patients. Hypophosphatemia has been linked to acute symptoms such as pain, nausea and muscle weakness, and more long term complications such as development of osteomalacia with bone pain, fractures and pseudo fractures. Transient hypophosphatemia 